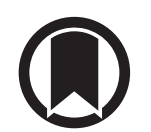

CrossMark

\title{
Standardised shorter regimens versus individualised longer regimens for rifampin- or multidrug-resistant tuberculosis
}

\author{
Syed Abidi ${ }^{1,2}$, Jay Achar $\mathbb{1}^{3}$, Mourtala Mohamed Assao Neino ${ }^{4}$, Didi Bang $\mathbb{C}^{5}$, \\ Andrea Benedetti ${ }^{1,2,6}$, Sarah Brode ${ }^{7}$, Jonathon R. Campbell (10,2, Esther C. Casas ${ }^{8}$, \\ Francesca Conradie ${ }^{9}$, Gunta Dravniece ${ }^{10}$, Philipp du Cros $^{3,11}$, Dennis Falzon ${ }^{12}$, \\ Ernesto Jaramillo ${ }^{12}$, Christopher Kuaban ${ }^{13}$, Zhiyi Lan ${ }^{1,2}$, Christoph Lange ${ }^{14,15,16,17}$, \\ Pei Zhi Li ${ }^{2}$, Mavluda Makhmudova ${ }^{18}$, Aung Kya Jai Maug ${ }^{19}$, Dick Menzies ${ }^{1,2}$, \\ Giovanni Battista Migliori (1020, Ann Miller (1)21, Bakyt Myrzaliev²2, \\ Norbert Ndjeka ${ }^{23}$, Jürgen Noeske ${ }^{24}$, Nargiza Parpieva ${ }^{25}$, Alberto Piubello ${ }^{19,26}$, \\ Valérie Schwoebel ${ }^{26}$, Welile Sikhondze 27 , Rupak Singla ${ }^{28}$, \\ Mahamadou Bassirou Souleymane ${ }^{19}$, Arnaud Trébucq ${ }^{26}$, Armand Van Deun ${ }^{29}$, \\ Kerri Viney ${ }^{30,31,32}$, Karin Weyer ${ }^{12}$, Betty Jingxuan Zhang ${ }^{1,2}$ and Faiz Ahmad Khan ${ }^{1,2}$
}

@ERSpublications

Standardised shorter regimens for RR/MDR-TB had substantially lower risk of loss to follow-up than individualised longer regimens, but also higher risk of failure or relapse if there was resistance to component drugs http://bit.ly/2RQgXzq

Cite this article as: Abidi S, Achar J, Assao Neino MM, et al. Standardised shorter regimens versus individualised longer regimens for rifampin- or multidrug-resistant tuberculosis. Eur Respir J 2020; 55: 1901467 [https://doi.org/10.1183/13993003.01467-2019].

ABSTRACT We sought to compare the effectiveness of two World Health Organization (WHO)recommended regimens for the treatment of rifampin- or multidrug-resistant (RR/MDR) tuberculosis (TB): a standardised regimen of 9-12 months (the "shorter regimen") and individualised regimens of $\geqslant 20$ months ("longer regimens").

We collected individual patient data from observational studies identified through systematic reviews and a public call for data. We included patients meeting WHO eligibility criteria for the shorter regimen: not previously treated with second-line drugs, and with fluoroquinolone- and second-line injectable agentsusceptible RR/MDR-TB. We used propensity score matched, mixed effects meta-regression to calculate adjusted odds ratios and adjusted risk differences (aRDs) for failure or relapse, death within 12 months of treatment initiation and loss to follow-up.

We included 2625 out of $3378(77.7 \%)$ individuals from nine studies of shorter regimens and 2717 out of 13104 (20.7\%) individuals from 53 studies of longer regimens. Treatment success was higher with the shorter regimen than with longer regimens (pooled proportions $80.0 \%$ versus $75.3 \%$ ), due to less loss to follow-up with the former ( $\mathrm{aRD}-0.15,95 \% \mathrm{CI}-0.17--0.12$ ). The risk difference for failure or relapse was slightly higher with the shorter regimen overall (aRD 0.02, 95\% CI 0-0.05) and greater in magnitude with baseline resistance to pyrazinamide ( $\mathrm{aRD} 0.12$, 95\% CI $0.07-0.16$ ), prothionamide/ethionamide ( $\mathrm{aRD}$ $0.07,95 \%$ CI $-0.01-0.16$ ) or ethambutol (aRD 0.09, 95\% CI 0.04-0.13).

In patients meeting WHO criteria for its use, the standardised shorter regimen was associated with substantially less loss to follow-up during treatment compared with individualised longer regimens and with more failure or relapse in the presence of resistance to component medications. Our findings support the need to improve access to reliable drug susceptibility testing.

This article has an editorial commentary: https://doi.org/10.1183/13993003.00224-2020

This article has supplementary material available from erj.ersjournals.com

Received: 23 July 2019 | Accepted after revision: 4 Dec 2019

The content of this work is copyright of the authors or their employers. Design and branding are copyright @ERS 2020. 


\section{Introduction}

Almost 600000 individuals develop tuberculosis (TB) disease caused by rifampin- or multidrug-resistant (RR/MDR) strains of Mycobacterium tuberculosis every year [1]. Treatment of RR/MDR-TB is challenging and these patients have a substantial risk of unfavourable outcomes [1].

Since 2016, World Health Organization (WHO) guidelines have included the option of treating RR/ MDR-TB with a standardised regimen of 9-12 months in duration (the "shorter regimen") instead of an individualised regimen of $\geqslant 20$ months ("longer regimens") [2]. Eligibility requirements for the shorter regimen include a high likelihood of susceptibility to fluoroquinolones and second-line injectable agents, and no previous treatment with second-line drugs. The shorter regimen is standardised; if any of its component drugs cannot be used then WHO recommends treatment with an individualised longer regimen. A number of uncertainties remain regarding these WHO recommendations.

First, the effectiveness of the shorter regimen compared with individualised longer regimens remains unclear. In a recently published randomised clinical trial comparing the shorter regimen with longer regimens composed per 2016 WHO guidelines, the shorter regimen was noninferior with respect to overall treatment success, but rates of nonconversion or reversion of cultures, relapse and death were higher in the shorter regimen arm $[3,4]$. These associations were not statistically significant, albeit the trial was not powered for each outcome. Second, because the shorter regimen is standardised, whether it is effective in the face of resistance to its component medications has remained a matter of debate [5-10]. The WHO recommendation against use of the shorter regimen in the presence of resistance to any of its component medications has been questioned as being too restrictive $[8,11]$. Third, it is unknown how the shorter regimen performs in comparison with longer regimens composed according to $2018 \mathrm{WHO}$ guidelines that recommend bedaquiline and linezolid, and discourage the use of second-line injectable agents [12].

In recent years, individual patient data meta-analyses from observational studies have tried to answer key questions about the treatment of RR/MDR-TB [13-15]. Considered the "gold standard" method for bringing together data from different studies, individual patient data meta-analysis includes a number of advantages over aggregate data meta-analysis. These include verification of data, standardisation of outcomes, use of multivariable analyses to adjust for potential confounding by other covariates and use of propensity score-based analyses to address potential confounding by indication [16, 17]. We applied this methodology to compare standardised shorter regimens to individualised regimens of longer duration.

\section{Methods}

\section{Objectives}

We sought to compare the effectiveness of standardised shorter regimens to regimens of longer duration, composed following WHO guidelines for the treatment of RR/MDR-TB.

Affiliations: ${ }^{1}$ McGill International TB Centre, Montreal, QC, Canada. ${ }^{2}$ Respiratory Epidemiology and Clinical Research Unit, Centre for Outcomes Research and Evaluation, McGill University and Research Institute of the McGill University Health Centre, Montreal, QC, Canada. ${ }^{3}$ Médecins Sans Frontières/Doctors without Borders, London, UK. ${ }^{4}$ National Tuberculosis Program, Niamey, Niger. ${ }^{5}$ International Reference Laboratory of Mycobacteriology, National Centre for Antimicrobials and Infection Control, Statens Serum Institut, Copenhagen, Denmark. ${ }^{6}$ Dept of Epidemiology, Biostatistics and Occupational Health, Faculty of Medicine, McGill University, Montreal, QC, Canada. ${ }^{7}$ West Park Healthcare Centre, University Health Network and University of Toronto, Toronto, ON, Canada. ${ }^{8}$ Médecins Sans Frontières/Doctors without Borders, Amsterdam, The Netherlands. ${ }^{9}$ Dept of Medicine, University of Witswatersrand, Johannesburg, South Africa. ${ }^{10} \mathrm{KNCV}$ TB Foundation, The Hague, The Netherlands. ${ }^{11}$ Burnet Institute, Melbourne, Australia. ${ }^{12}$ World Health Organization, Geneva, Switzerland. ${ }^{13}$ Faculty of Health Sciences, The University of Bamenda, Bambili, Cameroon. ${ }^{14}$ Research Center Borstel, Leibniz Lung Center, Borstel, Germany. ${ }^{15}$ German Center for Infection Research Clinical TB Unit, Borstel, Germany. ${ }^{16}$ Respiratory Medicine and International Health, University of Lübeck, Lübeck, Germany. ${ }^{17}$ Dept of Medicine, Karolinska Institute, Stockholm, Sweden. ${ }^{18} \mathrm{KNCV}$ Tajikistan, Dushanbe, Tajikistan. ${ }^{19}$ Damien Foundation, Brussels, Belgium. ${ }^{20}$ WHO Collaborating Centre for Tuberculosis and Lung Diseases, Istituti Clinici Scientifici Maugeri IRCCS, Tradate, Italy. ${ }^{21}$ Dept of Global Health and Social Medicine, Harvard Medical School, Boston, MA, USA. ${ }^{22}$ KNCV TB Foundation, Branch Office KNCV in Kyrgyzstan, Bishkek, Kyrgyzstan. ${ }^{23}$ National TB Programme, Republic of South Africa, Pretoria, South Africa. ${ }^{24}$ National Tuberculosis Programme, Yaounde, Cameroon. ${ }^{25}$ National TB Institute, Tashkent, Uzbekistan.

${ }^{26}$ International Union Against Tuberculosis and Lung Disease, Paris, France. ${ }^{27}$ National TB Control Program, Eswatini Ministry of Health, Mbabane, Swaziland. ${ }^{28}$ National Institute of Tuberculosis and Respiratory Diseases, Delhi, India. ${ }^{29}$ Mycobacteriology Unit, Institute of Tropical Medicine, Antwerp, Belgium. ${ }^{30}$ The University of Sydney, Sydney, Australia. ${ }^{31}$ Karolinska Institutet, Stockholm, Sweden. ${ }^{32}$ Australian National University, Canberra, Australia.

Correspondence: Faiz Ahmad Khan, 3D.60, 5252 Boulevard de Maisonneuve Ouest, Montreal, QC H4A 3S5, Canada. E-mail: faiz.ahmadkhandamcgill.ca 


\section{Regimen definitions}

We defined shorter regimens as standardised regimens with an intended duration of 9-12 months including 4-6 months of kanamycin, moxifloxacin, prothionamide, clofazimine, pyrazinamide, ethambutol and high-dose isoniazid, followed by 5-8 months of moxifloxacin, clofazimine, pyrazinamide, ethambutol and, optionally, prothionamide [2]. The following within-class drug substitutions were permitted: gatifloxacin or levofloxacin instead of moxifloxacin, ethionamide instead of prothionamide, amikacin or capreomycin instead of kanamycin and usual-dose isoniazid instead of high-dose isoniazid.

We defined longer regimens per 2016 WHO guidelines as individualised regimens that included a later-generation fluoroquinolone and a second-line injectable among at least five anti-TB medications considered to be effective based on drug susceptibility testing (DST) or at least four anti-TB medications considered effective plus pyrazinamide [2]. We counted bedaquiline, linezolid, carbapenems and delamanid as effective medications $[15,18]$. As WHO guidelines permit flexibility around the total recommended duration of 20 months [12], we used 18 months of treatment as the minimum total duration for a longer regimen.

In December 2018, WHO issued new guidelines for the composition of longer regimens. As such, we undertook an analysis (initially unplanned) to compare contemporary shorter and longer regimens. For this, we restricted shorter regimens to those using either moxifloxacin or levofloxacin (as gatifloxacin is no longer available) and we restricted longer regimens to those whose composition met 2018 WHO guidelines by using at least three drugs from group A (moxifloxacin/levofloxacin, bedaquiline, linezolid) plus at least one drug from group B (cycloserine/terizidone, clofazimine) or at least two drugs from each group, and not including kanamycin or capreomycin.

\section{Study selection, quality assessment and data management}

We identified studies from two previously published systematic reviews: one of shorter regimens [13] and one restricted to other regimens $[15,19]$. Search and selection criteria have been previously reported $[13$, 15, 19]. Briefly, we reviewed medical databases to identify studies of RR/MDR-TB treatment published from January 2009 to September 2015; the search was updated in April 2016. To be eligible, studies had to have reported end-of-treatment outcomes for at least 25 patients with bacteriologically confirmed RR/ MDR-TB, with clear descriptions of treatment regimens $[13,15,19]$. In this update, investigators of previously identified studies provided data on additional patients from their centres and we also added unpublished data that WHO had obtained through a public call for datasets issued in February 2018 [20]. We assessed study quality using a checklist of seven indicators adapted from the Risk Of Bias In Non-randomised Studies of Interventions (ROBINS-I) tool and classified studies into high, moderate or low quality $[15,21]$.

Investigators provided de-identified individual patient-level data on clinical variables (age, sex, HIV status, previous treatment with first- or second-line anti-TB drugs), methods and dates of TB diagnosis and DST, indicators of disease severity (results of sputum acid-fast bacilli microscopy, presence of cavities or bilateral involvement on chest radiographs), treatment regimen composition (including dose and duration for each drug), and end-of-treatment outcomes. We verified data with investigators, created variables common to all datasets and then concatenated the data to create two individual patient datasets: one for shorter regimens and one for longer regimens.

\section{Treatment outcomes}

Studies reported outcomes of cure, treatment completion, failure, loss to follow-up, death during treatment and relapse $[13,15]$. When defining these outcomes, the majority of longer regimen studies used 2008 or 2013 WHO definitions [22, 23] and shorter regimen studies used similar definitions adapted to a treatment duration of 9-12 months (supplementary table A1). For analysis, we combined outcomes of cure and treatment completion into a single outcome of treatment success. When studies provided data to distinguish re-infection and relapse, we only counted occurrences of the latter.

\section{Patient selection}

We included patients with RR/MDR-TB confirmed either by culture or molecular DST methods and meeting WHO criteria for use of the shorter standardised regimen: no previous treatment with second-line drugs and not infected with M. tuberculosis resistant to fluoroquinolones or second-line injectable agents (excluded by DST or considered unlikely). Patients with TB resistant to second-line injectable agents were included if treated with an alternative drug of the same class. We excluded individuals with DST-confirmed isoniazid-susceptible $\mathrm{TB}$ (i.e. the RR group consisted of individuals in whom DST to isoniazid had not been performed). 
From both longer and shorter regimen groups, we excluded patients who were not treated per WHO guidelines. Because WHO recommends the shorter regimen as standardised, we excluded patients in whom DST results had been used to alter the regimen's composition. From the longer regimen group, we excluded patients who did not receive a later-generation fluoroquinolone and a second-line injectable agent, and those treated with fewer than five effective drugs or with pyrazinamide and fewer than four effective drugs. We counted medications as effective if susceptibility was confirmed by DST, with the exception of cycloserine, clofazimine and linezolid, which we assumed effective in the absence of confirmed resistance, and bedaquiline, carbapenems and delamanid, which we always counted as effective. From both shorter and longer regimen groups, we excluded patients who had been assigned a successful treatment outcome but were treated for less than the minimal recommended duration (we used 8 and 17.5 months, respectively, as cut-offs for minimal duration). From the shorter regimen group, we excluded patients whose treatment was prolonged for $>1$ month beyond what their programme had reported as the maximum duration of shorter treatment; such exclusions did not apply to longer regimens because there is no recommended upper limit of treatment duration. We excluded patients with missing outcomes. Our mortality outcome was death during treatment, which meant that the likelihood of death being observed would be higher with lengthier durations of treatment. To avoid bias from this differential ascertainment, we excluded participants who died 12 months after starting therapy.

\section{Data analysis}

In all multivariable analyses, we adjusted for the following covariates that were considered important potential confounders: age, sex, HIV status, prior treatment with first-line anti-TB drugs and extensiveness of TB disease. We classified disease as extensive if sputum was smear positive or, when smear results were missing, if chest radiography demonstrated cavities. If cavitation was not reported, we classified disease as extensive if there were bilateral abnormalities on chest radiography.

We first calculated pooled percentages of each treatment outcome for shorter and longer regimens using random effects aggregate data meta-analyses with the exact binomial likelihood method [24]. Heterogeneity was estimated using the $I^{2}$ statistic. We then performed one-step individual patient-level data meta-analyses using generalised logistic mixed effects meta-regression to estimate adjusted odds ratios (aORs) (random intercept for matched pairs and fixed slope) and adjusted risk differences (aRDs) (fixed intercept and slope) with 95\% confidence intervals for the following outcomes: 1) failure or relapse versus success, 2) death versus success and 3) loss to follow-up versus success, failure or relapse. Estimates were calculated overall (including all patients) and within pre-specified subgroups, defined by HIV status, disease extensiveness, and baseline DST results for pyrazinamide, prothionamide/ethionamide and ethambutol. For analyses stratified by DST to these drugs, we excluded patients in whom fluoroquinolone susceptibility had been assumed rather than confirmed. We conducted two sensitivity analyses. In the first, we included patients with isoniazid-susceptible RR-TB. In the second, we compared the two regimens for the treatment of fluoroquinolone-resistant RR/MDR-TB.

We interpreted associations based on aORs rather than risk differences, as the former were estimated with random effects. Rather than using p-value-based decisions about statistical significance [25], we used the bounds of the $95 \%$ confidence intervals to determine if an association was potentially important. We considered a positive association (i.e. with OR point estimate $>1$ ) as important if the lower bound of the $95 \%$ confidence interval was $>0.95$ and a negative association (i.e. with OR point estimate $<1$ ) as important if the upper bound of the $95 \%$ confidence interval was $<1.05$ [25]. While we reported both aORs and aRDs in tables, in the text we refer to aRDs because risks are more intuitive to understand than odds.

In all analyses, adjustment was done using propensity scores that we calculated using the potential confounders. We matched shorter and longer regimen treated patients 1:1 with replacement [16], via the calliper method with difference of 0.02 allowed, and exact matching on HIV status. We imputed missing data with the method of multivariate imputation by chained equations for use in the adjusted analyses [26]. For calculating propensity scores, we imputed missing values for age, HIV status, prior use of first-line drugs and extensiveness of disease. We imputed DST for the purposes of counting the number of effective medications. We did not use imputed covariates or DST to select patients for subgroup analyses (e.g. if stratifying analyses by pyrazinamide resistance, we excluded patients without pyrazinamide DST). We generated 20 datasets that included measured and imputed values, performed multivariable analyses in each one, and then pooled the results using Rubin's rules to calculate adjusted effect estimates [26-28].

Meta-analyses and imputation were performed using the statistical software $\mathrm{R}$ with the packages metafor version 2.0-0, lme4 version 1.1-21 and mice version 3.4.0 [29, 30]. The protocol can be obtained by contacting the corresponding author. 
Role of the funding source

The WHO Global TB Programme funded the study and conducted the public call and collection of unpublished data. Employees of the Global TB Programme participated in data collection and analysis. The WHO Drug-Resistant TB Guidelines Development Group provided input on the statistical analysis plan, and reviewed and discussed our results when updating their guidelines in 2018. The corresponding author had access to all the data in the study and had final responsibility for the decision to submit for publication.

\section{Results}

\section{Study and patient selection}

We identified six studies of shorter regimens and 43 studies of longer regimens from previously published systematic reviews $[13,15,19]$, to which we added 13 studies (three shorter and 10 longer) identified through the WHO public call (supplementary figure A1). Individual-level data were available for 3378 patients treated with shorter regimens and 13104 patients treated with longer regimens. No important issues were identified in checking the individual patient data.

Table 1 enumerates the reasons why we excluded 753 out of 3378 (22\%) individuals treated with shorter regimens and 10387 out of 13104 (79\%) individuals treated with longer regimens. Of those excluded from the longer regimens, 5012 out of 10387 (48\%) were excluded because they did not meet WHO criteria for eligibility for shorter regimen treatment. Exclusions due to regimens whose composition did not follow WHO guidelines were more common in the longer regimen group, whereas exclusions due to treatment durations not meeting recommendations were more common in the shorter regimen group. The proportion of individuals excluded due to missing data on duration or outcomes was higher in the longer regimen group. In both groups, the proportion excluded due to deaths occurring after month 12 of treatment was small $(<1 \%)$. Overall, we included 2625 out of $3378(77.7 \%)$ individuals from nine studies

\begin{tabular}{|c|c|c|}
\hline & Shorter & Longer \\
\hline Patients in initial database & 3378 & 13104 \\
\hline \multicolumn{3}{|l|}{ Reasons for exclusion } \\
\hline Did not meet WHO criteria for standardised shorter regimens & 306 & 5012 \\
\hline Rifampin resistance not confirmed & 115 & 11 \\
\hline Previous treatment with second-line TB drugs & 33 & 2301 \\
\hline XDR-TB & 10 & 1912 \\
\hline Fluoroquinolone-resistant TB (excluding XDR) & 137 & 1149 \\
\hline Second-line injectable-resistant TB (excluding XDR) & 22 & 1222 \\
\hline Did not meet criteria for inclusion in individual patient data meta-analyses & 447 & 5375 \\
\hline Isoniazid-susceptible TB & 210 & 15 \\
\hline Not treated with a shorter regimen ${ }^{\#}$ & 151 & NA \\
\hline Not treated with later-generation fluoroquinolone & NA & 2954 \\
\hline Not treated with second-line injectable & NA & 917 \\
\hline Other & 23 & 0 \\
\hline Duration or outcome data missing & 52 & 1852 \\
\hline Not treated with $\geqslant 4$ effective drugs and pyrazinamide or $\geqslant 5$ effective drugs ${ }^{\pi}$ & NA & 775 \\
\hline $\begin{array}{l}\text { Successful outcome reported, but with less than minimum recommended } \\
\text { duration, or any outcome beyond maximum duration }{ }^{+}\end{array}$ & 52 & 154 \\
\hline Died after month 12 of treatment ${ }^{\S}$ & 2 & 121 \\
\hline Included in main analyses & 2625 & 2717 \\
\hline
\end{tabular}

Data are presented as $\mathrm{n}$. Individuals with more than one exclusion criteria are included in the counts for each applicable criterion, such that the sum of the exclusion criteria counts is greater than the total number of patients excluded. WHO: World Health Organization; TB: tuberculosis; XDR: extensively drug-resistant; NA: not applicable. " : we also excluded patients treated with standardised shorter treatment regimens modified to include a drug from a class outside of the usual composition le.g. para-aminosalicylic acid, cycloserine/terizidone, bedaquiline). ": among those otherwise meeting criteria for inclusion. ${ }^{+}$: we excluded patients in whom a successful outcome was recorded if their treatment duration was $<8$ months with a shorter regimen or $<17.5$ months with a longer regimen. Patients on shorter regimens were excluded regardless of their outcome if treatment lasted 1 month beyond the upper limit of the maximum duration of treatment with the shorter regimen. ${ }^{\S}$ : among those otherwise meeting criteria for inclusion. See Methods for rationale. 


\section{TABLE 2 Clinical characteristics of included patients}

\begin{tabular}{|c|c|c|}
\hline & Shorter & Longer \\
\hline Patients & 2625 & 2717 \\
\hline Age years & $35.4 \pm 13.0$ & $36.6 \pm 12.4$ \\
\hline Children and adolescents (age $<16$ years) & $53(2.0)$ & $29(1.1)$ \\
\hline Male & $1682(64.1)$ & $1590(58.5)$ \\
\hline People living with HIV & $380(14.5)$ & $1156(42.8)$ \\
\hline Antiretroviral therapy & $328(86.3)$ & $1077(93.2)$ \\
\hline Acid-fast bacilli smear positive & $2224(88.6)$ & $1820(69.4)$ \\
\hline Cavitation on chest radiography & $501(40.1)$ & 465 (52.2) \\
\hline Bilateral disease on chest radiography & $1617(88.6)$ & $409(61.6)$ \\
\hline Extensive disease $^{\#}$ & $2256(88.2)$ & $1873(69.1)$ \\
\hline Previous treatment with first-line drugs & $2209(87.7)$ & $1355(50.3)$ \\
\hline High-income country & $0(0.0)$ & $562(20.7)$ \\
\hline Upper-middle-income country & $41(1.6)$ & $1704(62.7)$ \\
\hline Low-middle- or low-income country & $2584(98.4)$ & $451(16.6)$ \\
\hline \multicolumn{3}{|l|}{ Pyrazinamide } \\
\hline Resistant & $317(52)$ & 440 (44.3) \\
\hline Sensitive & $293(48)$ & 554 (55.7) \\
\hline No data (\% of all) & $2015(76.8)$ & $1723(63.4)$ \\
\hline \multicolumn{3}{|l|}{ Ethambutol } \\
\hline Resistant & $843(63.9)$ & $723(62.5)$ \\
\hline Sensitive & $477(36.1)$ & 434 (37.5) \\
\hline No data (\% of all) & $1305(49.7)$ & $1560(57.4)$ \\
\hline \multicolumn{3}{|l|}{ Ethionamide/prothionamide } \\
\hline Resistant & $291(26.8)$ & $200(20.5)$ \\
\hline Sensitive & 795 (73.2) & 777 (79.5) \\
\hline No data $(\%$ of all) & $1539(58.6)$ & $1740(64.0)$ \\
\hline \multicolumn{3}{|l|}{ Clofazimine } \\
\hline Resistance & 0 & $4(5.3)$ \\
\hline Sensitive & $8(100)$ & 71 (94.7) \\
\hline No data $(\%$ of all $)$ & $2617(99.7)$ & 2642 (97.2) \\
\hline \multicolumn{3}{|l|}{ Para-aminosalicylic acid } \\
\hline Resistance & $10(1.5)$ & $57(7.0)$ \\
\hline Sensitive & 662 (98.5) & 756 (93.0) \\
\hline No data (\% of all) & $1953(74.4)$ & $1904(70.1)$ \\
\hline \multicolumn{3}{|l|}{ Cycloserine/terizidone } \\
\hline Resistance & & $16(2.8)$ \\
\hline Sensitive & & 549 (97.2) \\
\hline No data ( $\%$ of all) & $2625(100)$ & $2152(79.2)$ \\
\hline \multicolumn{3}{|l|}{ Linezolid } \\
\hline Resistance & & $2(1)$ \\
\hline Sensitive & & 190 (99) \\
\hline No data (\% of all) & $2625(100)$ & 2525 (92.9) \\
\hline
\end{tabular}

Data are presented as $\mathrm{n}$, mean \pm SD or $\mathrm{n}(\%)$, unless otherwise stated. ${ }^{\#}$ : patients were classified as having extensive disease if they were smear positive and as having disease that was not extensive if their sputum was smear negative; in those missing data on smear status, their disease was classified as extensive if chest radiographs demonstrated cavitation and not extensive in the absence of cavitation. In studies where cavitation was not reported, disease was classified as extensive if there were bilateral chest radiographic abnormalities and not extensive in the absence of bilateral involvement.

([7, 31-34] and unpublished data as indicated in the supplementary material) of shorter regimens and 2717 out of $13104(20.7 \%)$ individuals from 39 studies of longer regimens ([35-65] and unpublished data as indicated in the supplementary material). Of the 48 included studies, 39 (81\%) were of high quality, eight (17\%) were of moderate quality and one (2\%) was of low quality (supplementary table A2).

\section{Description of included patients and regimens}

Table 2 summarises patient characteristics and DST results. Distributions of age and sex were similar between the two groups. Patients treated with longer regimens were more likely to be people living with HIV and to have cavitary TB. Those treated with the shorter regimen were more likely to have smear-positive $\mathrm{TB}$, bilateral disease, previous treatment with first-line drugs and been treated in 


\begin{tabular}{lcc}
\multicolumn{2}{l}{ TABLE 3 Regimen composition of shorter and longer regimens included in analyses } & Longer \\
\hline & Shorter & 2717 \\
Patients in analysis & 2625 & $2444(90)$ \\
Drug used & & $1325(48.8)$ \\
Pyrazinamide & $2625(100)$ & $439(16.2)$ \\
Ethambutol & $2625(100)$ & $2131(78.4)$ \\
High-dose isoniazid & $2442(93)$ & $2(0.1)$ \\
Moxifloxacin & $1378(52.5)$ & $716(26.4)$ \\
Gatifloxacin & $1040(39.6)$ & $366(13.5)$ \\
Levofloxacin & $207(7.9)$ & $2032(74.8)$ \\
Amikacin & $21(0.8)$ & $476(17.5)$ \\
Kanamycin & $2471(94.1)$ & $2470(90.9)$ \\
Capreomycin & $135(5.1)$ & $167(6.1)$ \\
Prothionamide/ethionamide & $2625(100)$ & $244(9.0)$ \\
Clofazimine & $2625(100)$ & $825(30.4)$ \\
Linezolid & 0 & $901(33.2)$ \\
Para-aminosalicylic acid & 0 & $320(11.8)$ \\
Cycloserine & 0 & $21(0.7)$ \\
Bedaquiline & 0 & $16(0.6)$ \\
Carbapenems & 0 & $7.8(6.1-9.1)$ \\
Delamanid & 0 & $21.6(19.5-24.0)$ \\
Duration of intensive phase months &
\end{tabular}

Data are presented as $\mathrm{n}, \mathrm{n}(\%)$ or median (interquartile range). " : among successfully treated patients.

low-middle- or low-income countries (98.4\% versus $16.6 \%$ ). Numbers of people living with HIV not on antiretroviral therapy were small in both groups. Prevalence of resistance to pyrazinamide, prothionamide/ ethionamide and ethambutol was similar between the two groups. Resistance to pyrazinamide, ethambutol and prothionamide/ethionamide was correlated (supplementary table A3). Few patients had DST for clofazimine, para-aminosalicylic acid, cycloserine and linezolid.

Regimen composition is summarised in table 3. Moxifloxacin was the most common fluoroquinolone in both regimens. Only two out of $2717(0.1 \%)$ patients treated with longer regimens received gatifloxacin versus 1040 out of 2625 (39.6\%) patients treated with the shorter regimen. Kanamycin was the most common second-line injectable used. Less than half the patients treated with longer regimens received isoniazid, ethambutol and clofazimine versus all patients treated with the shorter regimen. In the longer regimen group, bedaquiline was used in 320 out of 2717 (11.8\%) patients, linezolid in 244 out of 2717 (9.0\%) patients, a carbapenem in 21 out of $2717(0.7 \%)$ patients and delamanid in 16 out of $2717(0.6 \%)$ patients.

Aggregate data meta-analyses

The pooled rate of treatment success was higher with the shorter regimen (80.0\%) compared with longer regimens $(75.3 \%)$ (table 4). Fewer patients treated with the shorter regimen were lost to follow-up (shorter $4.2 \%$ versus longer $14.6 \%$ ) and more experienced failure or relapse (shorter $3.6 \%$ versus longer $2.7 \%$ ) and death (shorter $7.6 \%$ versus longer $4.6 \%$ ). Heterogeneity was high for each outcome, for both regimens. Forest plots are given in supplementary figures A2-A4.

\section{Individual patient data meta-analyses}

In univariable analyses (supplementary table A4), failure or relapse was positively associated with extensive disease, ethambutol resistance and pyrazinamide resistance. Death was positively associated with age, HIV status and prior treatment with first-line drugs. Loss to follow-up was positively associated with male sex and prior treatment with first-line drugs, and negatively associated with pyrazinamide resistance.

In multivariable analyses, there was an association between treatment with the shorter regimen and higher odds and risks of failure or relapse that was borderline overall (table 5), but important in subgroups where there was baseline resistance to pyrazinamide (aRD 0.12, 95\% CI 0.07-0.16), prothionamide/ethionamide ( $\mathrm{aRD} 0.07,95 \% \mathrm{CI}-0.01-0.16$ ) and ethambutol ( $\mathrm{aRD} 0.09,95 \%$ CI $0.04-0.13$ ). In the presence of resistance to at least two of these medications, the shorter regimen was also associated with greater failure or relapse (aRD 0.10, 95\% CI 0.05-0.15) (supplementary table A5). Death during the first 12 months of 
TABLE 4 Pooled percentage of treatment outcomes from aggregate data meta-analysis

\begin{tabular}{ccccc} 
& Success & Failure or relapse & $\begin{array}{c}\text { Death during first } \\
\text { 12 months of treatment }\end{array}$ & Loss to follow-up \\
\hline Shorter (9 studies) & $2164 / 2625$ & $118 / 2625$ & $201 / 2625$ & $142 / 2625$ \\
Heterogeneity estimates & $80.0 \%(72.1-86.1 \%)$ & $3.6 \%(1.3-9.6 \%)$ & $7.6 \%(4.2-13.1 \%)$ & $4.2 \%(2.3-7.5 \%)$ \\
Longer (39 studies) & $I^{2}=92 \%, \tau^{2}=0.35$ & $I^{2}=95 \%, \tau^{2}=2.04$ & $I^{2}=91 \%, \tau^{2}=0.60$ & $I^{2}=85 \%, \tau^{2}=0.51$ \\
Heterogeneity estimates & $7814 / 2717$ & $112 / 2717$ & $265 / 2717$ & $526 / 2717$ \\
& $75.3 \%(69.8-80.0 \%)$ & $2.7 \%(1.5-4.7 \%)$ & $4.6 \%(2.9-7.2 \%)$ & $14.6 \%(11.0-19.0 \%)$ \\
& $I^{2}=79 \%, \tau^{2}=0.42$ & $I^{2}=60 \%, \tau^{2}=0.80$ & $I^{2}=69 \%, \tau^{2}=0.74$ & $I^{2}=76 \%, \tau^{2}=0.50$
\end{tabular}

treatment was not associated with regimen type (table 6). Risks of loss to follow-up were lower with the shorter regimen, overall ( $\mathrm{aRD}-0.15,95 \% \mathrm{CI}-0.17--0.12$ ) and in most subgroups (table 7).

A total of 1166 patients were included in the secondary analysis comparing moxifloxacin- or levofloxacin-containing shorter regimens $(n=1004)$ with longer regimens composed per 2018 WHO recommendations $(\mathrm{n}=162)$. As shown in supplementary table A6, the groups had similar distributions of age, sex and extensive disease. HIV infection was less common in the shorter regimen group (shorter $20.4 \%$ versus longer 57.4\%) and previous first-line treatment was more common (shorter $82.5 \%$ versus longer $45.9 \%$ ). Of patients treated with shorter regimens, $96 \%$ resided in low- or low-middle-income countries, whereas $92 \%$ of those treated with longer regimens resided in upper-middle-income countries. Resistance to pyrazinamide, ethambutol or prothionamide/ethionamide was each more common in the longer regimen group (pyrazinamide: shorter 59\% versus longer 77.3\%; ethambutol: shorter $67.6 \%$ versus longer 78.3\%; prothionamide/ethionamide: shorter $50.2 \%$ versus longer $61.9 \%$ ). As shown in table 8 , moxifloxacin- or levofloxacin-based shorter regimens were associated with greater risk of death compared with longer regimens constructed per $2018 \mathrm{WHO}$ guidelines, although confidence intervals included the null value (aOR 2.5, 95\% CI 1.0-6.3; aRD 0.11, 95\% CI $-0.01-0.22)$. There were no important differences in failure or relapse and loss to follow-up.

\section{Sensitivity analyses}

When patients with isoniazid-susceptible RR-TB were included (supplementary table A7a), failure or relapse was not associated with type of regimen, but death was weakly associated with the shorter regimen

TABLE 5 Comparison of shorter regimens and individualised longer regimens for outcome of failure or relapse versus success, using propensity score matched individual patient data meta-analysis

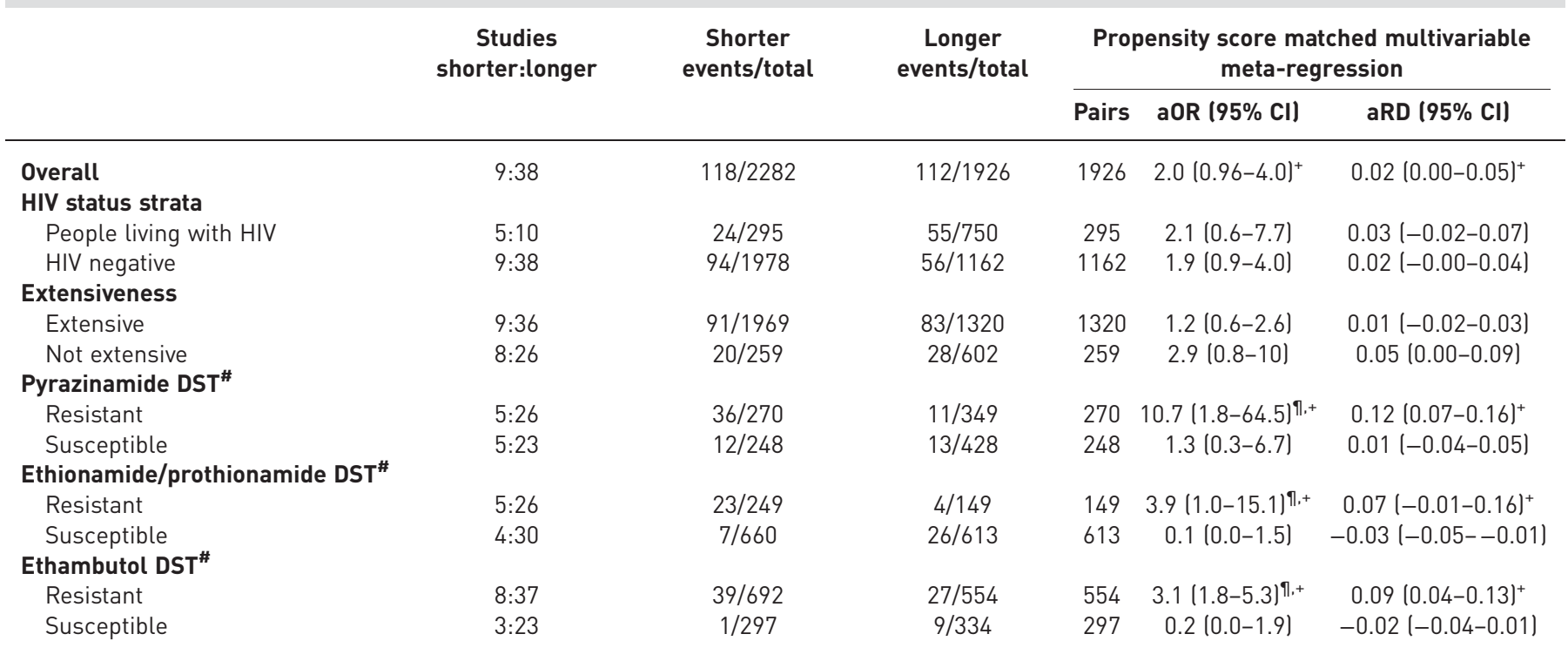

Data are presented as $n$, unless otherwise stated. aOR: adjusted odds ratio; aRD: adjusted risk difference; DST: drug susceptibility testing. All models adjusted for age, sex. HIV status, previous treatment with first-line tuberculosis medications and extensiveness of disease. Results were adjusted as described in the Methods. \#: analyses restricted to patients with DST-confirmed fluoroquinolone susceptibility (i.e. excluding those with no DST data for fluoroquinolones); ": aOR calculated from fixed effect model; ${ }^{+}$: confidence intervals suggestive of increased odds or risk of failure or relapse with the shorter regimen. 
TABLE 6 Comparison of shorter regimens and individualised longer regimens for outcome of death during the first 12 months of treatment versus success, using propensity score matched individual patient data meta-analysis

\begin{tabular}{|c|c|c|c|c|c|c|}
\hline & \multirow[t]{2}{*}{$\begin{array}{c}\text { Studies } \\
\text { shorter:longer }\end{array}$} & \multirow[t]{2}{*}{$\begin{array}{c}\text { Shorter } \\
\text { events/total }\end{array}$} & \multirow[t]{2}{*}{$\begin{array}{c}\text { Longer } \\
\text { events/total }\end{array}$} & \multicolumn{3}{|c|}{$\begin{array}{l}\text { Propensity score matched multivariable } \\
\text { meta-regression }\end{array}$} \\
\hline & & & & Pairs & aOR $(95 \% \mathrm{CI})$ & aRD $(95 \% \mathrm{CI})$ \\
\hline \multicolumn{7}{|l|}{ HIV status strata } \\
\hline People living with HIV & $5: 9$ & $72 / 343$ & $169 / 864$ & 343 & $1.0(0.6-1.6)$ & $0.00(-0.08-0.08)$ \\
\hline HIV negative & $9: 37$ & $127 / 2011$ & $96 / 1202$ & 1202 & $0.8(0.4-1.4)$ & $-0.01(-0.04-0.01)$ \\
\hline Not extensive & $8: 28$ & $27 / 266$ & $79 / 653$ & 266 & $1.6(0.5-5.6)$ & $0.02(-0.04-0.07)$ \\
\hline \multicolumn{7}{|l|}{ Pyrazinamide DST" } \\
\hline Resistant & $5: 27$ & $16 / 250$ & $33 / 371$ & 250 & $0.3(0.1-1.4)$ & $-0.05(-0.11-0.02)$ \\
\hline Susceptible & $4: 23$ & $19 / 255$ & 19/434 & 255 & $1.4(0.4-5.5)$ & $0.01(-0.05-0.07)$ \\
\hline \multicolumn{7}{|c|}{ Ethionamide/prothionamide DST" } \\
\hline Resistant & $4: 26$ & $18 / 244$ & $9 / 154$ & 154 & $1.5(0.3-7.4)$ & $0.02(-0.05-0.08)$ \\
\hline
\end{tabular}

Data are presented as $\mathrm{n}$, unless otherwise stated. aOR: adjusted odds ratio; aRD: adjusted risk difference; DST: drug susceptibility testing. All models adjusted for age, sex, HIV status, previous treatment with first-line tuberculosis medications and extensiveness of disease. Results were adjusted as described in the Methods. ${ }^{\#}$ : analyses restricted to patients with DST-confirmed fluoroquinolone susceptibility (i.e. excluding those with no DST data for fluoroquinolones).

(aOR 1.2, 95\% CI 0.96-1.5; aRD 0.02, 95\% CI -0.01-0.05). For RR/MDR-TB additionally resistant to fluoroquinolones, treatment with the shorter regimen was associated with increased failure or relapse (aOR 15.0, 95\% CI 2.8-80.6; aRD 0.33, 95\% CI 0.22-0.44) (supplementary table A7b).

TABLE 7 Comparison of shorter regimens and individualised longer regimens for outcome of loss to follow-up versus success, using propensity score matched individual patient data meta-analysis

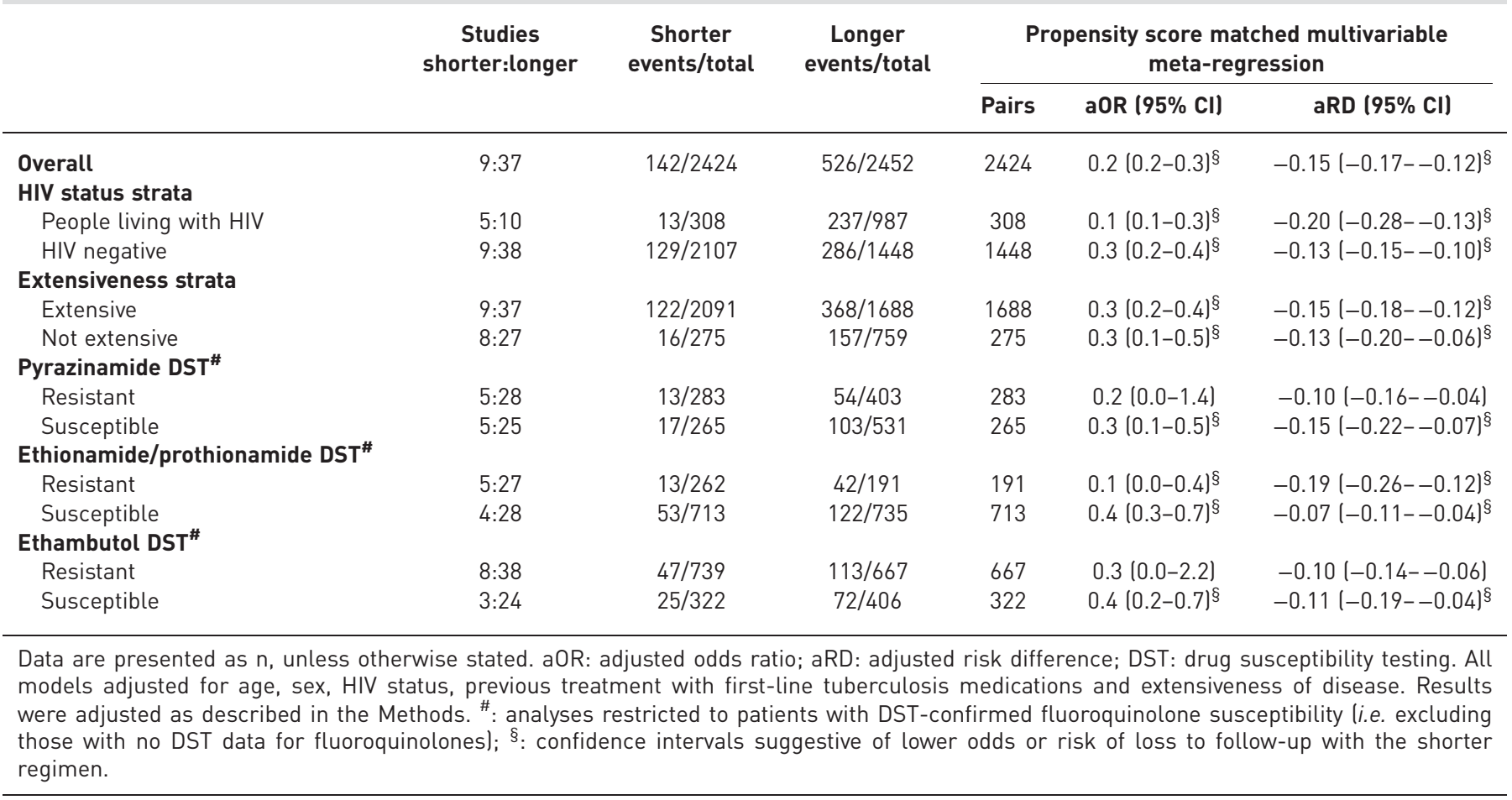


TABLE 8 Comparison of moxifloxacin- or levofloxacin-based shorter regimens to longer regimens meeting WHO 2018 composition and duration criteria, using propensity score matched individual patient data meta-analysis, among patients with rifampin- or multidrug-resistant tuberculosis (TB) confirmed susceptible to fluoroquinolones

\begin{tabular}{|c|c|c|c|c|c|c|}
\hline & \multirow[t]{2}{*}{$\begin{array}{c}\text { Studies } \\
\text { shorter:longer }\end{array}$} & \multirow[t]{2}{*}{$\begin{array}{c}\text { Shorter } \\
\text { events/total }\end{array}$} & \multirow[t]{2}{*}{$\begin{array}{c}\text { Longer } \\
\text { events/total }\end{array}$} & \multicolumn{3}{|c|}{$\begin{array}{l}\text { Propensity score matched multivariable } \\
\text { meta-regression }\end{array}$} \\
\hline & & & & Pairs & aOR $(95 \% \mathrm{CI})$ & $\operatorname{aRD}(95 \% \mathrm{CI})$ \\
\hline $\begin{array}{l}\text { Death during first } 12 \text { months of } \\
\text { treatment versus success }\end{array}$ & $9: 9$ & $79 / 879$ & $13 / 138$ & 138 & $2.5(1.0-6.3)^{+}$ & $0.11(-0.01-0.22)$ \\
\hline Lost versus success, failure or relapse & $9: 10$ & $44 / 925$ & $14 / 149$ & 149 & $0.6(0.1-4.5)$ & $-0.01(-0.09-0.07)$ \\
\hline
\end{tabular}

\section{Discussion}

In this individual patient-level data meta-analysis on the treatment of RR/MDR-TB without documented resistance to fluoroquinolones or second-line injectables, we found the unadjusted pooled rate of treatment success was higher with the standardised shorter regimen compared with individualised longer regimens composed per 2016 WHO guidelines. In adjusted analyses, we observed the standardised shorter regimen was associated with a higher risk of bacteriological failure or relapse, notably in the presence of resistance to pyrazinamide, prothionamide/ethionamide and ethambutol. We also observed that the adjusted risk of loss to follow-up while on treatment was lower among patients treated with the standardised shorter regimen, a finding consistent in multiple subgroup and sensitivity analyses. We did not identify significant associations between regimen and risk of death in our pre-specified analyses. A post hoc subgroup analysis comparing longer regimens that followed 2018 WHO guidelines (including bedaquiline and/or linezolid) with contemporary shorter regimens (that used either moxifloxacin or levofloxacin) found risk of death was significantly higher in the latter group.

Our findings are consistent with results of the STREAM study, the recently published randomised clinical trial that showed the noninferiority of the shorter regimen versus longer standardised regimens, for a composite end-point of bacteriological outcomes, death and treatment completion [3, 4]. In STREAM, the proportion not completing treatment per protocol was higher with the longer regimens $(30.3 \%$ versus $6.7 \%)$, there was a nonsignificant increase of unfavourable microbiological outcomes with the shorter regimen (relative risk of sputum culture nonconversion or reversion of 2.4, 95\% CI 0.85-7.0) [4] and, in the per protocol analysis, an unfavourable outcome was more likely with the shorter regimen in the presence of pyrazinamide resistance.

The findings from our study add to a growing body of evidence in support of increasing access to reliable and reproducible DST for all patients with RR/MDR-TB $[66,67]$. In a number of our analyses, resistance was associated with greater failure or relapse with the shorter regimen: for pyrazinamide, ethambutol, prothionamide/ethionamide as well as resistance to fluoroquinolones (assessed in a sensitivity analysis). However, there remains controversy about the clinical relevance of these findings [68-70], including among the authors of this study. This is because the association of failure or relapse with resistance to pyrazinamide, ethambutol or prothionamide/ethionamide in the treatment of RR/MDR-TB was not significant in some studies [7,31], and because concerns exist about the accuracy of DST to ethambutol and prothionamide/ethionamide [70-72].

Our study has a number of limitations. First, there is the possibility of bias from residual confounding, particularly because the majority of shorter regimen data originated from low- or low-middle-income countries (98.4\%) and the majority of longer regimen data originated from high- or upper-middle-income countries $(83.4 \%)$. Programmes in the latter settings are likely to have had greater resources, including for the management of comorbidities such as HIV, which would be expected to contribute to better outcomes. Second, it is possible that differences in the definition of treatment failure between longer and shorter regimen studies contributed to the observation of less failure with the former; however, our findings on failure and relapse were similar to those of STREAM where uniform outcome definitions were used. Third, the data available did not permit a comparison between shorter and longer regimen studies with respect to adverse events, due to important differences in ascertainment. However, in STREAM the frequency of grade $\geqslant 3$ adverse events was similar in the two arms $(45.4 \%$ and $48.2 \%$ for longer and shorter regimens, respectively) [3]. A recent meta-analysis restricted to longer regimens reported that risks of adverse events 
were low for bedaquiline, clofazimine and fluoroquinolones, and high for second-line injectables and linezolid [73]. Fourth, because we did not apply an upper limit to duration when defining longer regimens, it is possible that success rates with these regimens were underestimated due to confounding by indication for prolonged treatment. Fifth, because some patients lost to follow-up during treatment may have been undiagnosed failure cases, it is possible that failure or relapse was less likely to be detected in the longer regimens. Finally, caution is warranted in interpretation of differences in loss to follow-up as noncompletion of treatment with a shorter regimen may carry a greater risk of death or failure than noncompletion of a longer regimen. This was suggested in the STREAM trial, where even participants that did not complete treatment were followed, such that outcomes through 104 weeks of follow-up were known for $95 \%$ of those treated with the longer regimens and $99 \%$ of those treated with the shorter regimen. In that trial, excess deaths were observed in the latter group after week 76 of follow-up.

The study also has a number of strengths. First, the amount and quality of data, from a diversity of settings, has improved the generalisability and strengthened the evidence base for the shorter regimen. Second, individual-level data enabled us to reduce selection and confounding bias that could not have been addressed through aggregate meta-analysis. Third, we were able to contribute to the ongoing debate about the effectiveness of the standardised shorter regimen in the presence of resistance to component medications, something that was not fully addressed by the STREAM trial. Finally, we were able to compare the shorter regimen to longer regimens that follow 2018 WHO guidelines, an endeavour that would require a number of years if undertaken prospectively. However, our results should be interpreted with caution because this comparison was initially unplanned and based on a small subgroup.

\section{Conclusions}

Compared with individualised longer regimens for the treatment of RR/MDR-TB susceptible to fluoroquinolones and second-line injectables, the standardised shorter regimen is associated with less loss to follow-up. In the presence of resistance to pyrazinamide, ethambutol or prothionamide/ethionamide, the shorter regimen is associated with more failure or relapse. Our findings, and concerns about the reliability and reproducibility of phenotypic DST for some of these drugs, reinforce the need to increase access to reliable DST.

Author contributions: F. Ahmad Khan, D. Menzies, A. Benedetti, P. du Cros, J.R. Campbell, D. Falzon, Z. Lan, A. Piubello, V. Schwoebel and A. Trébucq designed the study and protocol. J. Achar, M.M. Assao Neino, A.K.J. Maug, D. Bang, S. Brode, E. Casas, F. Conradie, G. Dravniece, P. du Cros, C. Kuaban, A. Miller, B. Myrzaliev, G.B. Migliori, M. Makhmudova, J. Noeske, N. Ndjeka, A. Piubello, N. Parpieva, M.B. Souleymane, R. Singla, V. Schwoebel, W. Sikhondze and A. Trébucq contributed data to the meta-analysis. S. Abidi, F. Ahmad Khan, A. Benedetti, J.R. Campbell, P.Z. Li, Z. Lan, D. Menzies and B.J. Zhang did the data analysis. F. Ahmad Khan wrote the initial draft of the manuscript, and all authors provided critical input and revisions to the draft manuscripts, and approved the final manuscript.

Conflict of interest: S. Abidi has nothing to disclose. J. Achar has nothing to disclose. M.M. Assao Neino has nothing to disclose. D. Bang has nothing to disclose. A. Benedetti has nothing to disclose. S. Brode reports grants from Insmed and the Canadian Institutes for Health Research, personal fees for educational presentations from Boehringer Ingelheim and AstraZeneca, outside the submitted work. J.R. Campbell has nothing to disclose. E. Casas has nothing to disclose. F. Conradie has nothing to disclose. G. Dravniece has nothing to disclose. P. du Cros reports he was previously a member of the steering committee and protocol writing committee for the PRACTECAL randomised controlled trial of three novel 6-month MDR-TB regimens; and has undertaken a paid consultancy between TB Alliance and Burnet Institute to investigate applicability of the TB-Nix regimen (a novel short MDR-TB regimen) to Papua New Guinea. D. Falzon has nothing to disclose. E. Jaramillo has nothing to disclose. C. Kuaban has nothing to disclose. Z. Lan has nothing to disclose. C. Lange reports personal fees for lectures from Chiesi, Gilead, Janssen, Lucane, Novartis, Oxoid, Berlin-Chemie and Thermo Fisher, outside the submitted work. P.Z. Li has nothing to disclose. M. Makhmudova has nothing to disclose. A.K.J. Maug has nothing to disclose. D. Menzies has nothing to disclose. G.B. Migliori has nothing to disclose. A. Miller reports that The Eli Lilly Foundation MDR-TB Partnership provided partial salary support in 2015-2016 through a grant to Salmaan Keshavjee, Harvard Medical School, although none of the work in the current paper or analysis was supported through that mechanism; the grant also paid for travel to a meeting in July of 2016. B. Myrzaliev has nothing to disclose. N. Ndjeka has nothing to disclose. J. Noeske has nothing to disclose. N. Parpieva has nothing to disclose. A. Piubello has nothing to disclose. V. Schwoebel has nothing to disclose. W. Sikhondze has nothing to disclose. R. Singla has nothing to disclose. M.B. Souleymane has nothing to disclose. A. Trébucq has nothing to disclose. A. Van Deun has nothing to disclose. K. Viney has nothing to disclose. K. Weyer has nothing to disclose. B.J. Zhang has nothing to disclose. F. Ahmad Khan reports grants from the World Health Organization during the conduct of the study.

Support statement: This work was supported by the World Health Organization. Funding information for this article has been deposited with the Crossref Funder Registry.

\section{References}

$1 \quad$ World Health Organization. Global Tuberculosis Report 2017. Geneva, WHO, 2018.

2 World Health Organization. WHO Treatment Guidelines for Drug-resistant Tuberculosis - 2016 Update. Geneva, WHO, 2016. 
3 Nunn AJ, Phillips PPJ, Meredith SK, et al. A trial of a shorter regimen for rifampin-resistant tuberculosis. $N$ Engl J Med 2019; 380: 1201-1213.

4 World Health Organization. Position Statement on the Continued Use of the Shorter MDR-TB Regimen Following an Expedited Review of the STREAM Stage 1 Preliminary Results. Geneva, WHO, 2018.

5 Javaid A, Ahmad N, Khan AH, et al. Applicability of the World Health Organization recommended new shorter regimen in a multidrug-resistant tuberculosis high burden country. Eur Respir J 2017; 49: 1601967.

6 Rusen ID, Chiang CY. Building the evidence base for shortened MDR-TB treatment regimens. Int J Tuberc Lung Dis 2018; 22: 1-2.

7 Trebucq A, Schwoebel V, Kashongwe Z, et al. Treatment outcome with a short multidrug-resistant tuberculosis regimen in nine African countries. Int J Tuberc Lung Dis 2018; 22: 17-25.

8 Van Deun A, Chiang C-Y. Shortened multidrug-resistant tuberculosis regimens overcome low-level fluoroquinolone resistance. Eur Respir J 2017; 49: 1700223.

9 Varaine F, Guglielmetti L, Mitnick CD. Reply: Benefit of the shorter multidrug-resistant tuberculosis treatment regimen in California and modified eligibility criteria. Am J Respir Crit Care Med 2017; 196: 1490-1491.

10 Walsh KF, Souroutzidis A, Vilbrun SC, et al. Potentially high number of ineffective drugs with the standard shorter course regimen for multidrug-resistant tuberculosis treatment in Haiti. Am J Trop Med Hyg 2019; 100: 392-398.

11 Sotgiu G, Tiberi S, D'Ambrosio L, et al. Faster for less: the new "shorter" regimen for multidrug-resistant tuberculosis. Eur Respir J 2016; 48: 1503-1507.

12 World Health Organization. Rapid Communication: Key Changes to Treatment of Multidrug- and Rifampicin-resistant Tuberculosis (MDR/RR-TB). Geneva, WHO, 2018.

13 Ahmad Khan F, Salim MAH, du Cros P, et al. Effectiveness and safety of standardised shorter regimens for multidrug-resistant tuberculosis: individual patient data and aggregate data meta-analyses. Eur Respir J 2017; 50: 1700061.

14 Ahuja SD, Ashkin D, Avendano M, et al. Multidrug resistant pulmonary tuberculosis treatment regimens and patient outcomes: an individual patient data meta-analysis of 9,153 patients. PLoS Med 2012; 9: e1001300.

15 Collaborative Group for the Meta-Analysis of Individual Patient Data in MDRTB Treatment-2017. Treatment correlates of successful outcomes in pulmonary multidrug-resistant tuberculosis: an individual patient data meta-analysis. Lancet 2018; 392: 821-834.

16 Austin PC. An introduction to propensity score methods for reducing the effects of confounding in observational studies. Multivariate Behav Res 2011; 46: 399-424.

17 Stewart LA, Tierney JF. To IPD or not to IPD? Advantages and disadvantages of systematic reviews using individual patient data. Eval Health Prof 2002; 25: 76-97.

18 World Health Organization. The Use of Delamanid in the Treatment of Multidrug-resistant Tuberculosis in Children and Adolescents: Interim Policy Guidance. Geneva, WHO, 2016.

19 Bastos ML, Lan Z, Menzies D. An updated systematic review and meta-analysis for treatment of multidrug-resistant tuberculosis. Eur Respir J 2017; 49: 1600803.

20 World Health Organization. Public Call for Individual Patient Data on Treatment of Rifampicin and Multidrug-resistant (MDR/RR-TB) Tuberculosis. Geneva, WHO, 2018.

21 Sterne JA, Hernán MA, Reeves BC, et al. ROBINS-I: a tool for assessing risk of bias in non-randomised studies of interventions. BMJ 2016; 355: i4919.

22 World Health Organization. Definitions and Reporting Framework for Tuberculosis - 2013 Revision. Geneva, WHO, 2013

23 World Health Organization. Guidelines for the Programmatic Management of Drug-resistant Tuberculosis. Geneva, WHO, 2008.

24 Hamza TH, van Houwelingen HC, Stijnen T. The binomial distribution of meta-analysis was preferred to model within-study variability. J Clin Epidemiol 2008; 61: 41-51.

25 Wasserstein RL, Lazar NA. The ASA's statement on p-values: context, process, and purpose. Am Stat 2016; 70: $129-133$

26 Azur MJ, Stuart EA, Frangakis C, et al. Multiple imputation by chained equations: what is it and how does it work? Int J Methods Psychiatr Res 2011; 20: 40-49.

27 White IR, Royston P, Wood AM. Multiple imputation using chained equations: issues and guidance for practice. Stat Med 2011; 30: 377-399.

28 van Buuren S, Groothuis-Oudshoorn KJ. mice: multivariate imputation by chained equations in R. J Stat Softw 2011; 45: 1-67.

29 Bates D, Mächler M, Bolker B, et al. Fitting linear mixed-effects models using lme4. J Stat Softw 2015; 65: 1-48.

30 Viechtbauer WJ. Conducting meta-analyses in R with the metafor package. Stat Softw 2010; 36: 1-48.

31 Aung KJ, Van Deun A, Declercq E, et al. Successful "9-month Bangladesh regimen" for multidrug-resistant tuberculosis among over 500 consecutive patients. Int J Tuberc Lung Dis 2014; 18: 1180-1187.

32 Kuaban C, Noeske J, Rieder HL, et al. High effectiveness of a 12-month regimen for MDR-TB patients in Cameroon. Int J Tuberc Lung Dis 2015; 19: 517-524.

33 Piubello A, Harouna SH, Souleymane MB, et al. High cure rate with standardised short-course multidrug-resistant tuberculosis treatment in Niger: no relapses. Int J Tuberc Lung Dis 2014; 18: 1188-1194.

34 Van Deun A, Maug AK, Hamid Salim MA, et al. Short, highly effective, and inexpensive standardized treatment of multidrug-resistant tuberculosis. Am J Respir Crit Care Med 2010; 182: 684-692.

35 Anderson LF, Tamne S, Watson JP, et al. Treatment outcome of multi-drug resistant tuberculosis in the United Kingdom: retrospective-prospective cohort study from 2004 to 2007. Euro Surveill 2013; 18: 20601.

36 Anger HA, Dworkin F, Sharma S, et al. Linezolid use for treatment of multidrug-resistant and extensively drug-resistant tuberculosis, New York City, 2000-06. J Antimicrob Chemother 2010; 65: 775-783.

37 Bang D, Lillebaek T, Thomsen VØ, et al. Multidrug-resistant tuberculosis: treatment outcome in Denmark, 1992 2007. Scand J Infect Dis 2010; 42: 288-293.

38 Bonnet M, Pardini M, Meacci F, et al. Treatment of tuberculosis in a region with high drug resistance: outcomes, drug resistance amplification and re-infection. PLoS One 2011; 6: e23081.

39 Borisov SE, Dheda K, Enwerem M, et al. Effectiveness and safety of bedaquiline-containing regimens in the treatment of MDR- and XDR-TB: a multicentre study. Eur Respir J 2017; 49: 1700387. 
40 Cegielski JP, Kurbatova E, van der Walt M, et al. Multidrug-resistant tuberculosis treatment outcomes in relation to treatment and initial versus acquired second-line drug resistance. Clin Infect Dis 2016; 62: 418-430.

41 Chang KC, Leung CC, Yew WW, et al. Pyrazinamide may improve fluoroquinolone-based treatment of multidrug-resistant tuberculosis. Antimicrob Agents Chemother 2012; 56: 5465-5475.

42 Chang KC, Yew WW, Cheung SW, et al. Can intermittent dosing optimize prolonged linezolid treatment of difficult multidrug-resistant tuberculosis? Antimicrob Agents Chemother 2013; 57: 3445-3449.

43 Charles M, Vilbrun SC, Koenig SP, et al. Treatment outcomes for patients with multidrug-resistant tuberculosis in post-earthquake Port-au-Prince, Haiti. Am J Trop Med Hyg 2014; 91: 715-721.

44 Diacon AH, Pym A, Grobusch MP, et al. Multidrug-resistant tuberculosis and culture conversion with bedaquiline. $N$ Engl J Med 2014; 371: 723-732.

45 Eker B, Ortmann J, Migliori GB, et al. Multidrug- and extensively drug-resistant tuberculosis, Germany. Emerg Infect Dis 2008; 14: 1700-1706.

46 Guglielmetti L, Jaspard M, Le Du D, et al. Long-term outcome and safety of prolonged bedaquiline treatment for multidrug-resistant tuberculosis. Eur Respir J 2017; 49: 1601799.

47 Guglielmetti L, Le Du D, Jachym M, et al. Compassionate use of bedaquiline for the treatment of multidrug-resistant and extensively drug-resistant tuberculosis: interim analysis of a French cohort. Clin Infect Dis 2015; 60: 188-194.

48 Hughes $\mathrm{J}$, Isaakidis $\mathrm{P}$, Andries A, et al. Linezolid for multidrug-resistant tuberculosis in HIV-infected and -uninfected patients. Eur Respir J 2015; 46: 271-274.

49 Isaakidis $\mathrm{P}$, Varghese B, Mansoor H, et al. Adverse events among HIV/MDR-TB co-infected patients receiving antiretroviral and second line anti-TB treatment in Mumbai, India. PLoS One 2012; 7: e40781.

50 Jeong BH, Jeon K, Park HY, et al. Outcomes of pulmonary MDR-TB: impacts of fluoroquinolone resistance and linezolid treatment. J Antimicrob Chemother 2015; 70: 3127-3133.

51 Jo KW, Lee SD, Kim WS, et al. Treatment outcomes and moxifloxacin susceptibility in ofloxacin-resistant multidrug-resistant tuberculosis. Int J Tuberc Lung Dis 2014; 18: 39-43.

52 Kempker RR, Kipiani M, Mirtskhulava V, et al. Acquired drug resistance in Mycobacterium tuberculosis and poor outcomes among patients with multidrug-resistant tuberculosis. Emerg Infect Dis 2015; 21: 992-1001.

53 Koh WJ, Kang YR, Jeon K, et al. Daily $300 \mathrm{mg}$ dose of linezolid for multidrug-resistant and extensively drug-resistant tuberculosis: updated analysis of 51 patients. J Antimicrob Chemother 2012; 67: 1503-1507.

54 Kwak N, Kim HR, Yoo CG, et al. Changes in treatment outcomes of multidrug-resistant tuberculosis. Int J Tuberc Lung Dis 2015; 19: 525-530.

55 Laniado-Laborin R, Estrada-Guzman J, Perez $\mathrm{H}$, et al. Treatment of multidrug-resistant tuberculosis in a high-prevalence region through a binational consortium. Int J Tuberc Lung Dis 2012; 16: 610-611.

56 Marks SM, Flood J, Seaworth B, et al. Treatment practices, outcomes, and costs of multidrug-resistant and extensively drug-resistant tuberculosis, United States, 2005-2007. Emerg Infect Dis 2014; 20: 812-821.

57 Milanov V, Falzon D, Zamfirova M, et al. Factors associated with treatment success and death in cases with multidrug-resistant tuberculosis in Bulgaria, 2009-2010. Int J Mycobacteriol 2015; 4: 131-137.

58 Ndjeka N, Conradie F, Schnippel K, et al. Treatment of drug-resistant tuberculosis with bedaquiline in a high HIV prevalence setting: an interim cohort analysis. Int J Tuberc Lung Dis 2015; 19: 979-985.

59 Podewils LJ, Gler MT, Quelapio MI, et al. Patterns of treatment interruption among patients with multidrug-resistant TB (MDR TB) and association with interim and final treatment outcomes. PLoS One 2013; 8: e70064.

60 Pym AS, Diacon AH, Tang SJ, et al. Bedaquiline in the treatment of multidrug- and extensively drug-resistant tuberculosis. Eur Respir J 2016; 47: 564-574.

61 Smith SE, Ershova J, Vlasova N, et al. Risk factors for acquisition of drug resistance during multidrug-resistant tuberculosis treatment, Arkhangelsk Oblast, Russia, 2005-2010. Emerg Infect Dis 2015; 21: 1002-1011.

62 Tiberi S, Payen MC, Sotgiu G, et al. Effectiveness and safety of meropenem/clavulanate-containing regimens in the treatment of MDR- and XDR-TB. Eur Respir J 2016; 47: 1235-1243.

63 Tiberi S, Sotgiu G, D’Ambrosio L, et al. Comparison of effectiveness and safety of imipenem/clavulanate- versus meropenem/clavulanate-containing regimens in the treatment of MDR- and XDR-TB. Eur Respir J 2016; 47: $1758-1766$.

64 van Altena R, de Vries G, Haar CH, et al. Highly successful treatment outcome of multidrug-resistant tuberculosis in the Netherlands, 2000-2009. Int J Tuberc Lung Dis 2015; 19: 406-412.

65 Diacon AH, Pym A, Grobusch M, et al. The diarylquinoline TMC207 for multidrug-resistant tuberculosis. $N$ Engl J Med 2009; 360: 2397-2405.

66 Sun F, Li Y, Chen Y, et al. Introducing molecular testing of pyrazinamide susceptibility improves multidrug-resistant tuberculosis treatment outcomes: a prospective cohort study. Eur Respir J 2019; 53: 1801770.

67 Bastos ML, Hussain H, Weyer K, et al. Treatment outcomes of patients with multidrug-resistant and extensively drug-resistant tuberculosis according to drug susceptibility testing to first- and second-line drugs: an individual patient data meta-analysis. Clin Infect Dis 2014; 59: 1364-1374.

68 Yakrus MA, Driscoll J, McAlister A, et al. Molecular and growth-based drug susceptibility testing of Mycobacterium tuberculosis complex for ethambutol resistance in the United States. Tuberc Res Treat 2016; 2016: 3404860 .

69 Migliori GB, Sotgiu G, Rosales-Klintz S, et al. ERS/ECDC Statement: European Union standards for tuberculosis care, 2017 update. Eur Respir J 2018; 51: 1702678

70 World Health Organization. Technical Manual for Drug Susceptibility Testing of Medicines Used in the Treatment of Tuberculosis. Geneva, WHO, 2018.

71 Horne DJ, Pinto LM, Arentz M, et al. Diagnostic accuracy and reproducibility of WHO-endorsed phenotypic drug susceptibility testing methods for first-line and second-line antituberculosis drugs. J Clin Microbiol 2013; 51: 393-401.

72 Lakshmi R, Ramachandran R, Kumar DR, et al. Revisiting the susceptibility testing of Mycobacterium tuberculosis to ethionamide in solid culture medium. Indian J Med Res 2015; 142: 538-542.

73 Lan Z, Ahmad N, Baghaei P, et al. Drug-associated adverse events in the treatment of multidrug-resistant tuberculosis: an individual patient data meta-analysis. Lancet Respir Med 2019; in press. 